\title{
Malignant ascites with omental metastasis: a rare event in prostate cancer
}

\author{
Rajeev Saini, Chandragouda Dodagoudar, Vineet Talwar, Sajjan Singh \\ Department of Medical Oncology, Rajiv Gandhi Cancer Institute and Research Centre, New Delhi 110085, India.
}

Correspondence to: Dr. Rajeev Saini, Department of Medical Oncology, Rajiv Gandhi Cancer Institute and Research Centre, Sector 5, Rohini, New Delhi 110085, India. E-mail: rajeev.rajeev7@gmail.com

\section{A B S T RA C T}

Prostate cancer is the most common type of male malignancy in the world and approximately $10-20 \%$ of prostate cancer shows a metastatic disease at initial diagnosis commonly to the bones, vertebrae, ribs, long bones, and skull. However, prostate cancer metastasis to the omentum with malignant ascites is extremely uncommon. In this study, we report such a case, which also highlights a repeatedly negative ascetic fluid cytology even with multiple omental metastatic nodules. The purpose of this case report is to provide awareness to physicians for this rare occurrence.

Key words: Immunohistochemical staining, malignant ascites, omental metastasis, prostate cancer, prostatic specific antigen

\section{Introduction}

Prostate cancer is the most common male malignancy in the world with an estimated 1,100,000 new cases and 307,000 cancer-related deaths in 2012. ${ }^{[1]}$ Although most prostate cancer patients have localized disease with a favorable prognosis, advanced prostatic cancer metastasize frequently to the bone and regional lymph nodes, but prostate cancer metastasis to the omentum with malignant ascites is very rare. ${ }^{[2]}$ In this study, we report such a rare case.

\section{Case Report}

A 65-year-old man was initially diagnosed as prostate adenocarcinoma with Gleason score $7(4+3=7$ out 10) in 2004. The level of prostatic specific antigen (PSA) was $233 \mathrm{ng} / \mathrm{mL}$ at cancer diagnosis. He then underwent bilateral orchiectomy and hormonal therapy with $50 \mathrm{mg}$ dose of bicalutamide, but discontinued after 5 months treatment. In 2009, his PSA level raised to $90 \mathrm{ng} / \mathrm{mL}$, but there was no evidence of metastasis detected by either computed tomography (CT) or bone scan. He was again on bicalutamide treatment, but his PSA response lasted for approximately 2 years. In December 2011, bicalutamide treatment was discontinued, and Fosfestrol was started. However, in November 2013, his PSA level was increasing to $27.4 \mathrm{ng} / \mathrm{mL}$ and therefore, fosfestrol was discontinued and the patients were treated with ketoconazole and prednisolone.

\begin{tabular}{|l|l|}
\hline \multicolumn{2}{|c|}{ Access this article online } \\
\hline Quick Response Code: & Website: \\
\hline & www.jcmtjournal.com \\
\cline { 2 - 2 } & \\
\hline
\end{tabular}

One month after this regime of treatment, patient presented with 10 days history of abdominal distension and found to have gross ascites. A diagnostic and therapeutic paracentesis was conducted and removed 1,500 $\mathrm{mL}$ straw colored fluid. Fluid analysis showed to be exudate and cytology was negative for malignant cells. Ascitic fluid adenodeaminse titer and polymerase chain reaction showed negative for tuberculosis. Ascetic fluid was taken and tested for multiple times, but all were negative. Moreover, esophageal-gastrodudenoscopy and colonoscopy were normal. Contrast-enhanced CT abdomen in March 2014 showed prostatic mass with gross ascites with thickened omentum [Figure 1]. Bone scan shows no evidence of skeletal metastasis. Serum and ascitic PSA were $316 \mathrm{ng} / \mathrm{mL}$ and $175 \mathrm{ng} / \mathrm{mL}$, respectively. A ultrasound-guided biopsy of the thickened omentum and histology showed a metastatic adenocarcinoma [Figure 2], which was immunohistochemically positive for cytokeratin (CK) and PSA [Figure 3] and focally positive for CK7, whereas negative for CK2. Patient was then planned for Taxotere-based chemotherapy.

\section{Discussion}

Although prostate cancer can metastasize to nearly any organs in the body, metastasis without osseous involvement is extremely rare. Arnheim showed in 1948 that in 176 postmortem cases, the bone, lymph nodes, and lungs were the most common metastasis of prostate cancer, ${ }^{[2]}$ whereas the uncommon metastasis sites included the adrenal gland, kidney, brain, pancreas, genitalia, and breast. Malignant effusion, whether pleural or peritoneal, was an extremely rare. ${ }^{[2]}$ Moreover, Rapoport and Omenn ${ }^{[3]}$ reviewed the autopsy of 523 prostate cancer cases and found that 13 cases had peritoneal deposits, but with no other metastasis elsewhere in the body, 


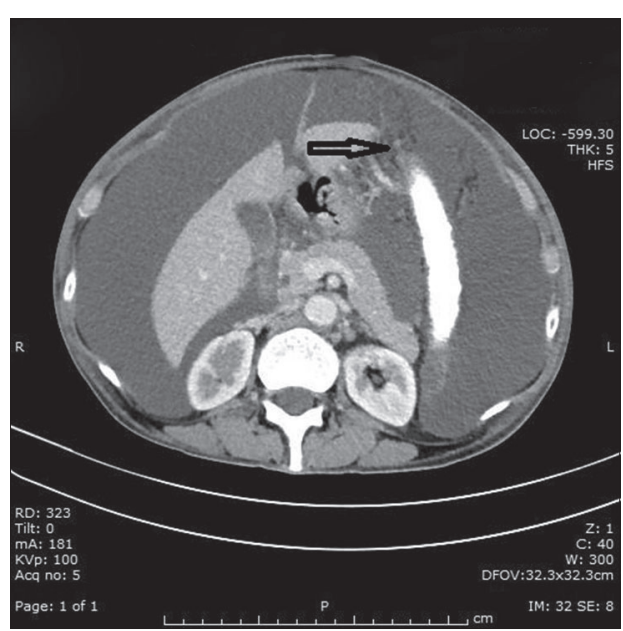

Figure 1: The computed tomography (CT) scan. The CT data show diffuse nodular thickening (black arrow) of the omentum and ascites

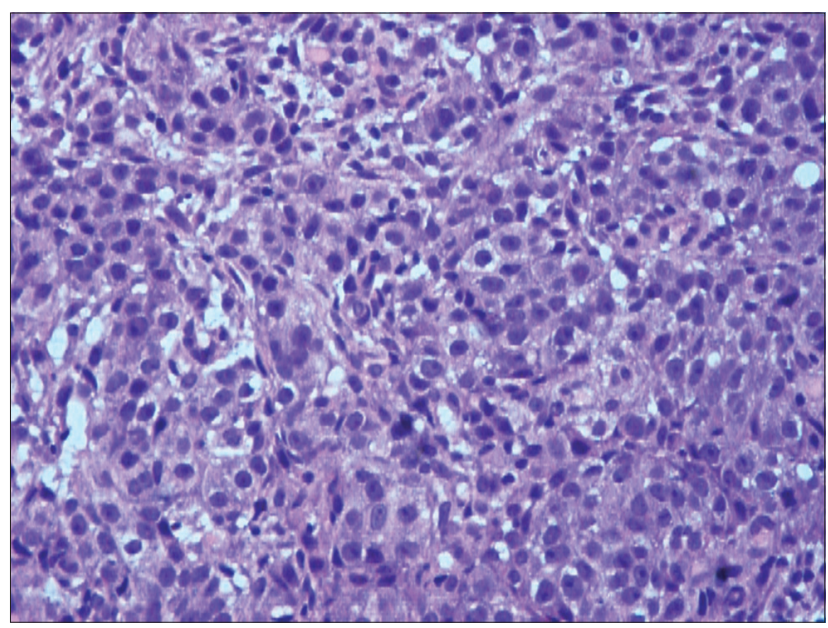

Figure 2: Hematoxylin and eosin staining of the omental biopsy Tissue section shows omental tissue infiltrated by poorly differentiated adenocarcinoma

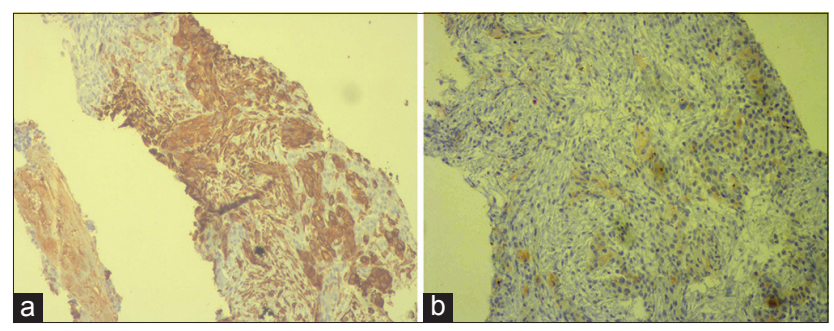

Figure 3: (a) Immunohistochemical staining of tissue biopsy for pan-cytokeratin (b) prostatic specific antigen

indicating that there were effusions occurring in prostate cancer patients without any involvements of the more common metastatic sites. Thus, if other benign (like tuberculosis in India) and malignant (especially gastrointestinal) $)^{[4]}$ etiologies should be excluded, these patients could reasonably be thought to be due to prostate cancer-induced ascites. Until date, there have been only 16 published cases of malignant ascites in prostate cancer ${ }^{[5]}$ and most cases presenting with malignant ascites were associated with other metastatic sites, including the bone, lymph nodes, omentum, rectal wall, liver, adrenal, and pleural effusions. ${ }^{[6]}$

The mechanism of malignant ascites may include peritoneal seedlings or lymphatic obstruction. Tumor cells in an effusion may have exfoliated from the primary lesion as evidenced by the positive cytology after repeated cytological examinations of ascetic fluid. However, negative cytology could be very difficult to make a differential diagnosis between benign and malignant ascites, such as the current case. The immunohistochemical staining can provide a valuable adjunction. For example, immunostaining of prostatic acid phosphatase and/or prostate specific antigen could be useful in the diagnosis of prostate cancer with a malignant effusion. ${ }^{[7]}$ Usually, malignant effusions in prostate cancer patients are associated with very poorer prognosis. ${ }^{[8]}$

\section{References}

1. Humphrey PA. Cancers of the male reproductive organs. In: Stewart BW, Wild CP, editors. World Cancer Report. Lyon: World Health Organization; 2014. p. 453-64.

2. Arnheim FK. Carcinoma of the prostate: a study of the postmortem findings in 176 cases. J Urol 1948;60:599-603.

3. Rapoport AH, Omenn GS. Dermatomyositis and malignant effusions: Rare manifestations of carcinoma of the prostate. J Urol 1968;100:183-7.

4. Megalli MR, Gursel EO, Veenema RJ. Ascites as an unusual presentation of carcinoma of the prostate. J Urol 1973;110:232-4.

5. Ani I, Costaldi M, Abouassaly R. Metastatic prostate cancer with malignant ascites: A case report and literature review. Can Urol Assoc J 2013;7:E248-50.

6. Saif MW. Malignant ascites associated with carcinoma of the prostate. J Appl Res 2005;5:305-11.

7. Satz N, Joller-Jemelka HI, Grob PJ, Hofer C, Schmid E, Knoblauch M. Tumor markers and immunomodulator substances in ascites -- their value as screening and diagnosis parameters. Schweiz Med Wochenschr 1989;119:762-5.

8. Kehinde EO, Abdeen SM, Al-Hunayan A, Ali Y. Prostate cancer metastatic to the omentum. Scand J Urol Nephrol 2002;36:225-7. 